\title{
Some fractional integral inequalities of type Hermite-Hadamard through convexity
}

\author{
Shahid Qaisar ${ }^{1}$, Jamshed Nasir², Saad Ihsan Butt², Asma Asma' , Farooq Ahmad ${ }^{3,4^{*}}$ (D), \\ Muhammad lqbal ${ }^{5}$ and Sajjad Hussain ${ }^{3,4}$
}

*Correspondence:
ahmad.farooq@ntu.edu.sg;
farooqgujar@gmail.com
3Present address: School of
Mechanical and Aerospace
Engineering, NANYANG
Technological University, Singapore,
Singapore
${ }^{4}$ Punjab Higher Education
Department (HED), Lahore, Pakistan
Full list of author information is
available at the end of the article

\begin{abstract}
In the present article, the authors have established some Hermite-Hadamard type integral inequalities via Riemann-Liouville fractional integrals that generalize Hermite-Hadamard type inequalities and a few other results (Dragomir and Agarwal in Appl. Math. Lett. 11(5):91-95, 1998; Dragomir, Chob and Kimc in J. Math. Anal. Appl. 245(2):489-501, 2000; Yang, Hwang and Tseng in Comput. Math. Appl.

47(2-3):207-216, 2004).
\end{abstract}

MSC: $26 \mathrm{~A} 15 ; 26 \mathrm{~A} 51 ; 26 \mathrm{D} 10$

Keywords: Hermite-Hadamard's inequality; Convex functions; Power-mean inequality; Riemann-Liouville fractional integration

\section{Introduction}

A function $g: I \subset \mathbb{R} \rightarrow \mathbb{R}$ is called convex in the classical sense, if the inequality

$$
g(\omega x+(1-\omega) y) \leq \omega g(x)+(1-\omega) g(y)
$$

holds for all $x, y \in I$ and $\omega \in[0,1]$. In fact a large number of articles have been written on inequalities using classical convexity but one of the most important and well known is Hermite-Hadamard's inequality, This double inequality is stated as follows [4]. Let $g: I \subset$ $\mathbb{R} \rightarrow \mathbb{R}$ be a convex function on the interval $I$ of real numbers and $x, y \in I$ with $x<y$. Then

$$
g\left(\frac{x+y}{2}\right) \leq \frac{1}{y-x} \int_{x}^{y} g(t) d t \leq \frac{g(x)+g(y)}{2} .
$$

Both inequalities hold in the reversed direction for $g$ to be concave. Several improvements and extensions of Hermite-Hadamard's type inequality to different kinds of convexity were established by different researchers.

First we recall some important definitions and results which we have used in this paper.

Definition 1 For $g \in L_{1}[a, b]$. The left-sided and right-sided Riemann-Liouville fractional integrals of order $\alpha>0$ with $a \geq 0$ are defined by

$$
J_{a^{+}}^{\alpha} g(x)=\frac{1}{\Gamma(\alpha)} \int_{a}^{x}(x-t)^{\alpha-1} g(t) d t, \quad a<x,
$$

(c) The Author(s) 2019. This article is distributed under the terms of the Creative Commons Attribution 4.0 International License (http://creativecommons.org/licenses/by/4.0/), which permits unrestricted use, distribution, and reproduction in any medium, provided you give appropriate credit to the original author(s) and the source, provide a link to the Creative Commons license, and indicate if changes were made. 
and

$$
J_{b^{-}}^{\alpha} g(x)=\frac{1}{\Gamma(\alpha)} \int_{x}^{b}(t-x)^{\alpha-1} g(t) d t, \quad x<b
$$

respectively, where $\Gamma(\cdot)$ is Gamma function and its definition is $\Gamma(\alpha)=\int_{0}^{\infty} e^{-u} u^{\alpha-1} d u$. It is to be noted that $J_{a^{+}}^{0} g(x)=J_{b^{-}}^{0} g(x)=g(x)$.

In the case of $\alpha=1$, the fractional integral reduces to the classical integral.

Properties relating to this operator can be found in [5] and for useful details on HermiteHadamard type inequalities connected with fractional integral inequalities, we refer the reader to [5-17] and the references therein.

In [18] Dragomir and Agarwal, obtained inequalities for differentiable convex mappings which are connected with the right-hand side of Hermite-Hadamard's (trapezoid) inequality and applied them to obtain some elementary inequalities for real numbers and in numerical integration as follows.

Theorem 1 Let $g: I \subset R \rightarrow R$ be a differentiable mapping on $I^{o}$ where $x, y \in I$ with $x<y$. If $\left|g^{\prime}\right|^{q}$ is convex on $[x, y]$, for some $q \geq 1$ then the following inequality holds:

$$
\left|\frac{g(x)+g(y)}{2}-\frac{1}{y-x} \int_{x}^{y} f(u) d u\right| \leq \frac{y-x}{8}\left[\left|g^{\prime}(x)\right|+\left|g^{\prime}(y)\right|\right]
$$

In [2] Dragomir, obtained inequalities for a Lipschitzian mapping which are in connection with the right-hand side of Hermite-Hadamard's (trapezoid) inequality.

Theorem 2 Let $g: I \subset R \rightarrow R$ be a M-Lipschitzian mapping on I where $x, y \in I$ with $x<y$, then we have the following inequality:

$$
\left|\frac{g(x)+g(y)}{2}-\frac{1}{y-x} \int_{x}^{y} f(u) d u\right| \leq \frac{M}{3}(y-x) .
$$

In [3] Yang, obtained Hermite-Hadamard's (trapezoid) inequalities for differentiable mapping for concave function.

Theorem 3 Let $I \subset \mathbb{R}$ be an open interval, $l, m, n, P, Q \in I$ with $l \leq P \leq n \leq Q \leq m(n \neq$ $l, m) l, m, n \in \mathbb{R}$ and $g:[x, y] \rightarrow \mathbb{R}$ be a differentiable function. If $\left|g^{\prime}\right|^{q}$ is concave on $[x, y]$ and $1 \leq \theta \leq q$, then

$$
\begin{aligned}
& \left|(P-l) g(l)+(m-Q) g(m)+(Q-P) g(n)-\int_{x}^{y} g(u) d u\right| \\
& \quad \leq K(P, Q, n, \theta) \cdot J(P, Q, n, \theta),
\end{aligned}
$$

where

$$
K(P, Q, n, \theta)=\left(\frac{1}{2}\left[(P-l)^{2}+(n-P)^{2}+(Q-n)^{2}+(m-Q)^{2}\right]\right)^{\frac{(\theta-1)}{\theta}}
$$


and

$$
\begin{aligned}
& J(P, Q, n, \theta) \\
& =\left(\frac{1}{2}\left[(P-l)^{2}+(n-P)^{2}\right]\left|g^{\prime}\left(\frac{(P-l)^{2}+(n-P)^{2}(2 n-3 l+P)}{3\left[(P-l)^{2}+(n-P)^{2}\right]}+l\right)\right|^{\theta}\right)^{\frac{(\theta-1)}{\theta}} \\
& \quad+\left(\frac{1}{2}\left[(Q-n)^{2}+(m-Q)^{2}\right]\left|g^{\prime}\left(m-\frac{(Q-n)^{2}(3 m-2 n-Q)+(m-Q)^{3}}{3\left[(Q-n)^{2}+(m-Q)^{2}\right]}\right)\right|^{\theta}\right)^{\frac{(\theta-1)}{\theta}} .
\end{aligned}
$$

Corollary 1 Under the assumptions of Theorem 3 with $P=Q=n=(l+m) / 2$ and $\theta=1$, we get the following inequality:

$$
\left|\frac{g(x)+g(y)}{2}-\frac{1}{y-x} \int_{x}^{y} g(u) d u\right| \leq \frac{y-x}{8}\left[\left|g^{\prime}\left(\frac{5 x+y}{6}\right)\right|+\left|g^{\prime}\left(\frac{x+5 y}{6}\right)\right|\right] .
$$

The goal of this article is to establish Hermite-Hadamard type inequalities for the Riemann-Liouville fractional integral using convexity as well as concavity, for functions whose absolute values of the first derivative are convex. Here we will derive a general integral inequality for the Riemann-Liouville fractional integral.

\section{Main results}

Before going on our main result first we prove the following integral inequality.

Lemma 1 Let $I \subset \mathbb{R}$ be an open interval, $a, b \in I$ with $a<b$ and $f:[a, b] \rightarrow \mathbb{R}$ be $a$ differentiable function such that $f^{\prime}$ is integrable and $0<\alpha \leq 1$ on $(a, b)$ with $a<b$. If $\left|f^{\prime}\right|$ is convex on $[a, b]$, then we have the following inequality:

$$
\begin{aligned}
& {\left[\left(\frac{(b-a)^{\alpha}-(x-a)^{\alpha}}{(b-a)^{\alpha}}+\frac{(b-x)^{\alpha}}{(b-a)^{\alpha}}\right) \frac{f(b)}{2}+\left(\frac{(b-a)^{\alpha}-(b-x)^{\alpha}}{(b-a)^{\alpha}}+\frac{(x-a)^{\alpha}}{(b-a)^{\alpha}}\right) \frac{f(a)}{2}\right.} \\
& \left.\quad-\frac{\Gamma(\alpha+1)}{2(b-a)^{\alpha}}\left[J_{a^{+}}^{\alpha} f(b)+J_{b^{-}}^{\alpha} f(a)\right]=\frac{1}{2} \sum_{k=1}^{4} I_{1 k}\right],
\end{aligned}
$$

where

$$
\begin{aligned}
& I_{11}=\frac{(x-a)^{\alpha+1}}{(b-a)^{\alpha}} \int_{0}^{1}\left(t^{\alpha}-1\right) f^{\prime}(t x+(1-t) a) d t, \\
& I_{12}=\frac{(b-x)^{\alpha+1}}{(b-a)^{\alpha}} \int_{0}^{1}\left(1-t^{\alpha}\right) f^{\prime}(t x+(1-t) b) d t, \\
& I_{13}=\frac{(b-x)^{\alpha+1}}{(b-a)^{\alpha}} \int_{0}^{1}\left[\left(\frac{a-b}{x-b}-t\right)^{\alpha}-\left(\frac{a-x}{x-b}\right)^{\alpha}\right] f^{\prime}(t x+(1-t) b) d t, \\
& I_{14}=\frac{(x-a)^{\alpha+1}}{(b-a)^{\alpha}} \int_{0}^{1}\left[\left(\frac{b-x}{x-a}\right)^{\alpha}-\left(\frac{b-a}{x-a}-t\right)^{\alpha}\right] f^{\prime}(t x+(1-t) a) d t .
\end{aligned}
$$

Proof Integrating by parts

$$
I_{11}=\frac{(x-a)^{\alpha+1}}{(b-a)^{\alpha}} \int_{0}^{1}\left(t^{\alpha}-1\right) f^{\prime}(t x+(1-t) a) d t
$$




$$
\begin{aligned}
= & \frac{(x-a)^{\alpha+1}}{(b-a)^{\alpha}}\left\{\left.\frac{\left(t^{\alpha}-1\right) f(t x+(1-t) a)}{x-a}\right|_{0} ^{1}\right. \\
& \left.+\frac{\alpha}{x-a} \int_{0}^{1}\left(t^{\alpha-1}\right) f(t x+(1-t) a) d t\right\} \\
= & \frac{(x-a)^{\alpha+1}}{(b-a)^{\alpha}}\left\{\frac{f(a)}{x-a}-\frac{\alpha}{x-a} \int_{a}^{x} \frac{(u-a)^{\alpha-1}}{(x-a)^{\alpha-1}} \cdot \frac{f(u) d u}{(x-a)}\right\} \\
= & \frac{f(a)(x-a)^{\alpha}}{(b-a)^{\alpha}}-\frac{\alpha}{(b-a)^{\alpha}} \int_{a}^{x}(u-a)^{\alpha-1} f(u) d u, \\
I_{13}= & \frac{(b-x)^{\alpha+1}}{(b-a)^{\alpha}} \int_{0}^{1}\left[\left(\frac{a-b}{x-b}-t\right)^{\alpha}-\left(\frac{a-x}{x-b}\right)^{\alpha}\right] f^{\prime}(t x+(1-t) b) d t \\
= & \left.\frac{(b-x)^{\alpha+1}}{(b-a)^{\alpha}} \frac{\left[\left(\frac{a-b}{x-b}-t\right)^{\alpha}-\left(\frac{a-x}{x-b}\right)^{\alpha}\right] f(t x+(1-t) b) d t}{x-b}\right|_{0} ^{1} \\
& -\int_{0}^{1}-\alpha\left(\frac{a-b}{x-b}-t\right)^{\alpha-1} \frac{f(t x+(1-t) b) d t}{x-b} \\
= & \frac{(b-x)^{\alpha+1}}{(b-a)^{\alpha}}\left[\left(\frac{a-b}{x-b}-t\right)^{\alpha}-\left(\frac{a-x}{b-x}\right)^{\alpha}\right] \frac{f(b)}{b-x} \\
& +\frac{-\alpha}{b-x} \int_{b}^{x} \frac{(u-a)^{\alpha-1}}{(b-x)^{\alpha-1}} \cdot \frac{f(u) d u}{(b-x)} \\
= & \frac{(b-x)^{\alpha+1}}{(b-a)^{\alpha}}\left\{\frac{(b-a)^{\alpha}-(x-a)^{\alpha}}{(b-x)^{\alpha+1}} f(b)-\frac{\alpha}{(b-x)^{\alpha+1}} \int_{x}^{b}(u-a)^{\alpha-1} f(u) d u\right\} .
\end{aligned}
$$

Analogously

$$
\begin{aligned}
& I_{12}=\frac{f(b)(b-x)^{\alpha}}{(b-a)^{\alpha}}-\frac{\alpha}{(b-a)^{\alpha}} \int_{b}^{x}(b-u)^{\alpha-1} f(u) d u, \\
& I_{14}=\frac{(x-a)^{\alpha+1}}{(b-a)^{\alpha}}\left\{\frac{-(b-x)^{\alpha}+(b-a)^{\alpha}}{(x-a)^{\alpha+1}} f(a)-\frac{\alpha}{(x-a)^{\alpha+1}} \int_{a}^{x}(b-u)^{\alpha-1} f(u) d u\right\} .
\end{aligned}
$$

Adding the above equalities, we get

$$
\begin{aligned}
& I_{11}+I_{13}=\frac{(x-a)^{\alpha}}{(b-a)^{\alpha}} f(a)+\left(1-\frac{(x-a)^{\alpha}}{(b-a)^{\alpha}}\right) f(b)-\frac{\Gamma(\alpha+1)}{(b-a)^{\alpha}} J_{b^{-}}^{\alpha} f(a), \\
& I_{12}+I_{14}=\frac{(b-x)^{\alpha}}{(b-a)^{\alpha}} f(b)+\left(1-\frac{(b-x)^{\alpha}}{(b-a)^{\alpha}}\right) f(a)-\frac{\Gamma(\alpha+1)}{(b-a)^{\alpha}} J_{a^{+}}^{\alpha} f(b) .
\end{aligned}
$$

The proof is completed.

Theorem 4 Let $I \subset \mathbb{R}$ be an open interval, $a, b \in I$ with $a<b$ and $f:[a, b] \rightarrow \mathbb{R}$ be $a$ differentiable function such that $f^{\prime}$ is integrable and $0<\alpha \leq 1$ on $(a, b)$ with $a<b$. If $\left|f^{\prime}\right|$ is convex on $[a, b]$, then the following inequality for Riemann-Liouville fractional integrals holds:

$$
\begin{aligned}
& {\left[\left(\frac{(b-a)^{\alpha}-(x-a)^{\alpha}}{(b-a)^{\alpha}}+\frac{(b-x)^{\alpha}}{(b-a)^{\alpha}}\right) \frac{f(b)}{2}+\left(\frac{(b-a)^{\alpha}-(b-x)^{\alpha}}{(b-a)^{\alpha}}+\frac{(x-a)^{\alpha}}{(b-a)^{\alpha}}\right) \frac{f(a)}{2}\right.} \\
& \left.-\frac{\Gamma(\alpha+1)}{2(b-a)^{\alpha}}\left[J_{a^{+}}^{\alpha} f(b)+J_{b^{-}}^{\alpha} f(a)\right]\right]
\end{aligned}
$$




$$
\begin{aligned}
\leq & \frac{(x-a)^{\alpha+1}}{(b-a)^{\alpha+1}}\left[A\left|f^{\prime}(x)\right|+B\left|f^{\prime}(a)\right|\right]+\frac{(b-x)^{\alpha+1}}{(b-a)^{\alpha+1}}\left[A\left|f^{\prime}(x)\right|+B\left|f^{\prime}(b)\right|\right] \\
& +\frac{(b-x)^{\alpha+1}}{(b-a)^{\alpha+1}}\left[C\left|f^{\prime}(x)\right|+D\left|f^{\prime}(b)\right|\right]+\frac{(x-a)^{\alpha+1}}{(b-a)^{\alpha+1}}\left[E\left|f^{\prime}(x)\right|+F\left|f^{\prime}(a)\right|\right]
\end{aligned}
$$

where

$$
\begin{aligned}
A= & \int_{0}^{1}\left|1-t^{\alpha}\right| t d t=\frac{\alpha}{2(\alpha+2)}, \\
B= & \int_{0}^{1}\left|1-t^{\alpha}\right|(1-t) d t=\frac{\alpha}{\alpha+1}-\frac{\alpha}{2(\alpha+2)}=\frac{\alpha(\alpha+3)}{2(\alpha+1)(\alpha+2)}, \\
C= & \int_{0}^{1} t\left|\left(\frac{a-b}{x-b}-t\right)^{\alpha}-\left(\frac{a-x}{x-b}\right)^{\alpha}\right| d t \\
= & -\frac{1}{(\alpha+1)(\alpha+2)}\left(\frac{a-x}{x-b}\right)^{\alpha+2}+\frac{1}{2}\left(\frac{a-x}{x-b}\right) \\
& +\frac{1}{(\alpha+1)(\alpha+2)}\left(\frac{a-b}{x-b}\right)^{\alpha+2}-\frac{1}{\alpha+1}\left(\frac{a-x}{x-b}\right)^{\alpha+1}-\left(\frac{a-x}{x-b}\right)^{\alpha}, \\
D= & \int_{0}^{1}\left|\left(\frac{a-b}{x-b}-t\right)^{\alpha}-\left(\frac{a-x}{x-b}\right)^{\alpha}\right|(1-t) d t \\
= & \frac{1}{(\alpha+1)}\left(\frac{a-b}{x-b}\right)^{\alpha+1}+\frac{1}{(\alpha+1)(\alpha+2)}\left(\frac{a-x}{x-b}\right)^{\alpha+2}-\frac{1}{2}\left(\frac{a-x}{x-b}\right) \\
& -\frac{1}{(\alpha+1)(\alpha+2)}\left(\frac{a-b}{x-b}\right)^{\alpha+2} \cdot
\end{aligned}
$$

Proof Here, utilizing the properties of the modulus in Lemma 1 and convexity of $\left|f^{\prime}\right|$, we have

$$
\begin{aligned}
\left|K_{1}\right| & =\frac{(x-a)^{\alpha+1}}{(b-a)^{\alpha}} \int_{0}^{1}\left(t^{\alpha}-1\right) f^{\prime}(t x+(1-t) a) d t, \\
\left|K_{1}\right| & \leq \frac{(x-a)^{\alpha+1}}{(b-a)^{\alpha}} \int_{0}^{1}\left|\left(1-t^{\alpha}\right)\right| f^{\prime}(t x+(1-t) a) \mid d t \\
& \leq \frac{(x-a)^{\alpha+1}}{(b-a)^{\alpha}} \int_{0}^{1}\left|\left(1-t^{\alpha}\right)\right|\left\{t\left|f^{\prime}(x)\right|+(1-t)\left|f^{\prime}(a)\right|\right\} d t, \\
\left|K_{1}\right| & =\frac{(x-a)^{\alpha+1}}{(b-a)^{\alpha}}\left\{A\left|f^{\prime}(x)\right|+B\left|f^{\prime}(a)\right|\right\},
\end{aligned}
$$

and analogously

$$
\begin{aligned}
\left|K_{2}\right| & =\frac{(b-x)^{\alpha+1}}{(b-a)^{\alpha}} \int_{0}^{1}\left(t^{\alpha}-1\right) f^{\prime}(t x+(1-t) b) d t, \\
\left|K_{2}\right| & \leq \frac{(b-x)^{\alpha+1}}{(b-a)^{\alpha}} \int_{0}^{1}\left|1-t^{\alpha}\right|\left|f^{\prime}(t x+(1-t) b)\right| d t \\
& \leq \frac{(b-x)^{\alpha+1}}{(b-a)^{\alpha}} \int_{0}^{1}\left|\left(1-t^{\alpha}\right)\right|\left\{t\left|f^{\prime}(x)\right|+(1-t)\left|f^{\prime}(b)\right|\right\} d t, \\
\left|K_{2}\right| & =\frac{(b-x)^{\alpha+1}}{(b-a)^{\alpha}}\left\{A\left|f^{\prime}(x)\right|+B\left|f^{\prime}(b)\right|\right\},
\end{aligned}
$$


using the convexity on $\left|f^{\prime}\right|$ and the fact that, for $\alpha \in(0,1]$ and $\forall t \in[0,1]$,

$$
\begin{aligned}
\left|K_{3}\right| & \leq \frac{(b-x)^{\alpha+1}}{(b-a)^{\alpha}} \int_{0}^{1}\left|\left(\frac{a-b}{x-b}-t\right)^{\alpha}-\left(\frac{a-x}{x-b}\right)^{\alpha}\right|\left|f^{\prime}(t x+(1-t) b)\right| d t \\
& \leq \frac{(b-x)^{\alpha+1}}{(b-a)^{\alpha}} \int_{0}^{1}\left|\left(\frac{a-b}{x-b}-t\right)^{\alpha}-\left(\frac{a-x}{x-b}\right)^{\alpha}\right|\left\{t\left|f^{\prime}(x)\right|+(1-t)\left|f^{\prime}(b)\right|\right\} d t, \\
\left|K_{3}\right| & =\frac{(b-x)^{\alpha+1}}{(b-a)^{\alpha}}\left\{C\left|f^{\prime}(x)\right|+D\left|f^{\prime}(b)\right|\right\},
\end{aligned}
$$

and analogously

$$
\begin{aligned}
\left|K_{4}\right| & \leq \frac{(x-a)^{\alpha+1}}{(b-a)^{\alpha}} \int_{0}^{1}\left|\left(\frac{b-x}{x-a}\right)^{\alpha}-\left(\frac{b-a}{x-a}-t\right)^{\alpha}\right|\left|f^{\prime}(t x+(1-t) a)\right| d t \\
& \leq \frac{(x-a)^{\alpha+1}}{(b-a)^{\alpha}} \int_{0}^{1}\left|\left(\frac{b-x}{x-a}\right)^{\alpha}-\left(\frac{b-a}{x-a}-t\right)^{\alpha}\right|\left\{t\left|f^{\prime}(x)\right|+(1-t)\left|f^{\prime}(a)\right|\right\} d t \\
& \leq \frac{(x-a)^{\alpha+1}}{(b-a)^{\alpha}}\left\{E\left|f^{\prime}(x)\right|+F\left|f^{\prime}(a)\right|\right\} .
\end{aligned}
$$

The proof is completed.

Remark 1 On letting $\alpha=1, x=\frac{a+b}{2}$ in Theorem 4 , inequality (5) reduces to inequality (1).

Theorem 5 Let $I \subset \mathbb{R}$ be an open interval, $a, b \in I$ with $a<b$ and $f:[a, b] \rightarrow \mathbb{R}$ be $a$ differentiable function such that $f^{\prime}$ is integrable and $0<\alpha \leq 1$ on $(a, b)$ with $a<b$. If $\left|f^{\prime}\right|^{q}$ is convex on $[a, b], q \geq 1$ then the following inequality holds:

$$
\begin{aligned}
& {\left[\left(\frac{(b-a)^{\alpha}-(x-a)^{\alpha}}{(b-a)^{\alpha}}+\frac{(b-x)^{\alpha}}{(b-a)^{\alpha}}\right) \frac{f(b)}{2}+\left(\frac{(b-a)^{\alpha}-(b-x)^{\alpha}}{(b-a)^{\alpha}}+\frac{(x-a)^{\alpha}}{(b-a)^{\alpha}}\right) \frac{f(a)}{2}\right.} \\
& \left.\quad+-\frac{\Gamma(\alpha+1)}{2(b-a)^{\alpha}}\left[J_{a^{+}}^{\alpha} f(b)+J_{b^{-}}^{\alpha} f(a)\right]\right] \\
& \leq\left[\frac{(x-a)^{\alpha+1}}{(b-a)^{\alpha}}\left(\gamma_{1}\right)^{1-1 / q}\left(A\left|f^{\prime}(x)\right|^{q}+B\left|f^{\prime}(a)\right|^{q}\right)^{1 / q}\right. \\
& \quad+\frac{(b-x)^{\alpha+1}}{(b-a)^{\alpha}}\left(\gamma_{2}\right)^{1-1 / q}\left(A\left|f^{\prime}(x)\right|^{q}+B\left|f^{\prime}(b)\right|^{q}\right)^{1 / q} \\
& \quad+\frac{(b-x)^{\alpha+1}}{(b-a)^{\alpha}}\left(\gamma_{3}\right)^{1-1 / q}\left(C\left|f^{\prime}(x)\right|^{q}+D\left|f^{\prime}(b)\right|^{q}\right)^{1 / q} \\
& \left.\quad+\frac{(x-a)^{\alpha+1}}{(b-a)^{\alpha}}\left(\gamma_{4}\right)^{1-1 / q}\left(E\left|f^{\prime}(x)\right|^{q}+F\left|f^{\prime}(a)\right|^{q}\right)^{1 / q}\right],
\end{aligned}
$$

where

$$
\begin{aligned}
E= & \int_{0}^{1} t\left|\left(\frac{b-a}{x-a}-t\right)^{\alpha}-\left(\frac{b-x}{x-a}\right)^{\alpha}\right| d t \\
= & \left(\frac{b-x}{2(x-a)}\right)-\frac{1}{(\alpha+1)}\left(\frac{b-x}{x-a}\right)^{\alpha+1}-\frac{b-x}{(x-a)} \\
& -\frac{1}{(\alpha+1)(\alpha+2)}\left(\frac{b-x}{x-a}\right)^{\alpha+2}+\frac{1}{(\alpha+1)(\alpha+2)}\left(\frac{b-a}{x-a}\right)^{\alpha+2},
\end{aligned}
$$


Qaisar et al. Journal of Inequalities and Applications

(2019) 2019:111

Page 7 of 10

$$
\begin{aligned}
F & =\int_{0}^{1}\left|\left(\frac{b-a}{x-a}-t\right)^{\alpha}-\left(\frac{b-x}{x-a}\right)^{\alpha}\right|(1-t) d t \\
& =\frac{1}{(\alpha+1)}\left(\frac{b-a}{x-a}\right)^{\alpha+2}-\frac{b-x}{2(x-a)}+\frac{1}{(\alpha+1)(\alpha+2)}\left(\frac{b-x}{x-a}\right)^{\alpha+2}-E \\
\gamma_{1} & =\int_{0}^{1}\left|t^{\alpha}-1\right| d t=\frac{\alpha}{(\alpha+1)}, \\
\gamma_{2} & =\int_{0}^{1}\left|1-t^{\alpha}\right| d t=\frac{\alpha}{(\alpha+1)}, \\
\gamma_{3} & =\int_{0}^{1}\left|\left(\frac{a-b}{x-b}-t\right)^{\alpha}-\left(\frac{a-x}{x-b}\right)^{\alpha}\right| d t \\
& =-\frac{1}{(\alpha+1)}\left(\frac{a-x}{x-b}\right)^{\alpha+1}-\left(\frac{a-x}{x-b}\right)^{\alpha}+\frac{1}{(\alpha+1)}\left(\frac{a-b}{x-b}\right)^{\alpha}, \\
\gamma_{4} & =\int_{0}^{1}\left|\left(\frac{b-x}{x-a}-t\right)^{\alpha}-\left(\frac{b-x}{x-a}\right)^{\alpha}\right| d t \\
& =\left(\frac{b-x}{x-a}\right)^{\alpha}+\frac{1}{(\alpha+1)}\left(\frac{b-x}{x-a}\right)^{\alpha+1}-\frac{1}{(\alpha+1)}\left(\frac{b-a}{x-a}\right)^{\alpha+1} .
\end{aligned}
$$

Proof By using the properties of the modulus in Lemma 1, we have

$$
\begin{aligned}
& \mid\left[\left(\frac{(b-a)^{\alpha}-(x-a)^{\alpha}}{(b-a)^{\alpha}}+\frac{(b-x)^{\alpha}}{(b-a)^{\alpha}}\right) \frac{f(b)}{2}+\left(\frac{(b-a)^{\alpha}-(b-x)^{\alpha}}{(b-a)^{\alpha}}+\frac{(x-a)^{\alpha}}{(b-a)^{\alpha}}\right) \frac{f(a)}{2}\right. \\
& \left.\quad+-\frac{\Gamma(\alpha+1)}{2(b-a)^{\alpha}}\left[J_{a^{+}}^{\alpha} f(b)+J_{b^{-}}^{\alpha} f(a)\right]\right] \leq \sum_{k=1}^{4}\left|J_{k}\right|
\end{aligned}
$$

and using convexity of $\left|f^{\prime}\right|$, we have

$$
\begin{aligned}
\left|J_{1}\right| & \leq \frac{(x-a)^{\alpha+1}}{(b-a)^{\alpha}} \int_{0}^{1}\left(1-t^{\alpha}\right)\left|f^{\prime}(t x+(1-t) a)\right| d t \\
& \leq \frac{(x-a)^{\alpha+1}}{(b-a)^{\alpha}}\left(\int_{0}^{1}\left(1-t^{\alpha}\right) d t\right)^{1-\frac{1}{q}}\left(\int_{0}^{1}\left(1-t^{\alpha}\right)\left|f^{\prime}(t x+(1-t) a)\right|^{q} d t\right)^{\frac{1}{q}} \\
& =\frac{(x-a)^{\alpha+1}}{(b-a)^{\alpha}}\left(\gamma_{1}\right)^{1-\frac{1}{q}}\left[A\left|f^{\prime}(x)\right|^{q}+B\left|f^{\prime}(a)\right|^{q}\right]^{\frac{1}{q}}
\end{aligned}
$$

and analogously

$$
\begin{aligned}
\left|J_{3}\right| \leq & \frac{(b-x)^{\alpha+1}}{(b-a)^{\alpha}} \int_{0}^{1}\left|\left(\frac{a-b}{x-b}-t\right)^{\alpha}-\left(\frac{a-x}{x-b}\right)^{\alpha}\right|\left|f^{\prime}(t x+(1-t) b)\right| d t \\
\leq & \frac{(b-x)^{\alpha+1}}{(b-a)^{\alpha}}\left(\int_{0}^{1}\left|\left(\frac{a-b}{x-b}-t\right)^{\alpha}-\left(\frac{a-x}{x-b}\right)^{\alpha}\right| d t\right)^{1-\frac{1}{q}} \\
& \times\left(\left.\int_{0}^{1}\left|\left(\frac{a-b}{x-b}-t\right)^{\alpha}-\left(\frac{a-x}{x-b}\right)^{\alpha}\right| f^{\prime}(t x+(1-t) b)\right|^{q} d t\right)^{\frac{1}{q}} \\
= & \frac{(b-x)^{\alpha+1}}{(b-a)^{\alpha}}\left(\gamma_{3}\right)^{1-\frac{1}{q}}\left[C\left|f^{\prime}(x)\right|^{q}+D\left|f^{\prime}(b)\right|^{q}\right]^{\frac{1}{q}},
\end{aligned}
$$


using the convexity and the fact that, for $\alpha \in(0,1]$ and $\forall t \in[0,1]$,

$$
\left|J_{2}\right| \leq \frac{(b-x)^{\alpha+1}}{(b-a)^{\alpha}}\left(\gamma_{2}\right)^{1-\frac{1}{q}}\left[A\left|f^{\prime}(x)\right|^{q}+B\left|f^{\prime}(b)\right|^{q}\right]^{\frac{1}{q}}
$$

and similarly

$$
\begin{aligned}
\left|J_{4}\right| \leq & \frac{(b-x)^{\alpha+1}}{(b-a)^{\alpha}} \int_{0}^{1}\left|\left(\frac{b-a}{x-a}-t\right)^{\alpha}-\left(\frac{b-x}{x-a}\right)^{\alpha}\right| f^{\prime}(t x+(1-t) a) \mid d t \\
\leq & \frac{(b-x)^{\alpha+1}}{(b-a)^{\alpha}}\left(\int_{0}^{1}\left|\left(\frac{b-a}{x-a}-t\right)^{\alpha}-\left(\frac{b-x}{x-a}\right)^{\alpha}\right| d t\right)^{1-\frac{1}{q}} \\
& \times\left(\int_{0}^{1}\left|\left(\frac{b-a}{x-a}-t\right)^{\alpha}-\left(\frac{b-x}{x-a}\right)^{\alpha}\right|\left|f^{\prime}(t x+(1-t) a)\right|^{q} d t\right)^{\frac{1}{q}} \\
= & \frac{(b-x)^{\alpha+1}}{(b-a)^{\alpha}}\left(\gamma_{4}\right)^{1-\frac{1}{q}}\left[E\left|f^{\prime}(x)\right|^{q}+F\left|f^{\prime}(a)\right|^{q}\right]^{\frac{1}{q}} .
\end{aligned}
$$

The proof is completed.

Corollary 2 On letting $\alpha=1, x=\frac{a+b}{2}$ and $\left|f^{\prime}(a)\right|=\left|f^{\prime}(b)\right| \leq M$ in Theorem 5, inequality (6) reduces to the inequality

$$
\left|\frac{f(a)+f(b)}{2}-\frac{1}{b-a} \int_{a}^{b} f(x) d x\right| \leq \frac{M}{4}(b-a) .
$$

Remark 2 The obtained inequality (7) is an improvement of the inequality as in (2).

In the following, we obtain an estimate of the Hermite-Hadamard inequality for concave functions.

Theorem 6 Let $f:[a, b] \rightarrow \mathbb{R}$ be a differentiable function on $(a, b)$ such that $f^{\prime} \in L_{1}[a, b]$. If $\left|f^{\prime}\right|^{q}$ is concave on $[a, b]$, for some fixed $p>1$ with $q=\frac{p}{p-1}$, the following inequality for fractional integrals holds:

$$
\begin{aligned}
& \mid\left[\left(\frac{(b-a)^{\alpha}-(x-a)^{\alpha}}{(b-a)^{\alpha}}+\frac{(b-x)^{\alpha}}{(b-a)^{\alpha}}\right) \frac{f(b)}{2}+\left(\frac{(b-a)^{\alpha}-(b-x)^{\alpha}}{(b-a)^{\alpha}}+\frac{(x-a)^{\alpha}}{(b-a)^{\alpha}}\right) \frac{f(a)}{2}\right. \\
&\left.\quad+-\frac{\Gamma(\alpha+1)}{2(b-a)^{\alpha}}\left[J_{a^{\prime}}^{\alpha} f(b)+J_{b-}^{\alpha} f(a)\right]\right] \mid \\
& \leq {\left[\left\{\gamma_{1}\left|f^{\prime}\left((\alpha+1)\left\{\frac{A x+B b}{\alpha}\right\}\right)\right|+\gamma_{2}\left|f^{\prime}\left((\alpha+1)\left\{\frac{A x+B b}{\alpha}\right\}\right)\right|\right\}\right.} \\
&\left.+\gamma_{3}\left|f^{\prime}\left((\alpha+1)\left\{\frac{C x+D b}{\alpha}\right\}\right)\right|+\gamma_{3}\left|f^{\prime}(\alpha+1)\left\{\frac{E x+F a}{\alpha}\right\}\right|\right] .
\end{aligned}
$$

Proof Using the concavity of $\left|f^{\prime}\right|^{q}$ and the power-mean inequality, we obtain

$$
\begin{aligned}
\left|f^{\prime}(t x+(1-t) y)\right|^{q} & >t\left|f^{\prime}(x)\right|^{q}+(1-t)\left|f^{\prime}(y)\right|^{q} \\
& \geq t\left|f^{\prime}(x)\right|^{q}+(1-t)\left|f^{\prime}(y)\right|^{q} .
\end{aligned}
$$


Hence

$$
\left|f^{\prime}(t x+(1-t) y)\right| \geq t\left|f^{\prime}(x)\right|+(1-t)\left|f^{\prime}(y)\right|
$$

so $\left|f^{\prime}\right|$ is also concave. By the Jensen integral inequality, we have

$$
\begin{aligned}
\left|I_{1}\right| & \leq \frac{(x-a)^{\alpha+1}}{(b-a)^{\alpha+1}}\left(\int_{0}^{1}\left|1-t^{\alpha}\right| d t\right)\left|f^{\prime}\left(\frac{\int_{0}^{1}\left|\left(1-t^{\alpha}\right)\right|\left[f^{\prime}(t x+(1-t) a)\right] d t}{\int_{0}^{1}\left|1-t^{\alpha}\right| d t}\right)\right| \\
& =\frac{(x-a)^{\alpha+1}}{(b-a)^{\alpha+1}}\left(\gamma_{1}\right)\left|f^{\prime}\left(\frac{A x+B a}{\gamma_{1}}\right)\right|
\end{aligned}
$$

and similarly

$$
\begin{aligned}
\left|I_{2}\right| \leq & \frac{(b-x)^{\alpha+1}}{(b-a)^{\alpha+1}}\left(\gamma_{2}\right)\left|f^{\prime}\left(\frac{A x+B b}{\gamma_{2}}\right)\right| \\
\left|I_{3}\right| \leq & \frac{(b-x)^{\alpha+1}}{(b-a)^{\alpha}}\left(\int_{0}^{1}\left|\left(\frac{a-b}{x-b}-t\right)^{\alpha}-\left(\frac{a-x}{x-b}\right)^{\alpha}\right|\right) \\
& \times\left|f^{\prime}\left(\frac{\left(\int_{0}^{1}\left|\left(\frac{a-b}{x-b}-t\right)^{\alpha}-\left(\frac{a-i x}{x-b}\right)^{\alpha}\right|\right)\left[f^{\prime}(t x+(1-t) b)\right] d t}{\int_{0}^{1}\left|\left(\frac{a-b}{x-b}-t\right)^{\alpha}-\left(\frac{a-x}{x-b}\right)^{\alpha}\right| d t}\right)\right| \\
\leq & \left(\gamma_{3}\right)\left|f^{\prime}\left(\frac{C x+D b}{\gamma_{3}}\right)\right|
\end{aligned}
$$

and

$$
\begin{aligned}
\left|I_{4}\right| \leq & \frac{(x-a)^{\alpha+1}}{(b-a)^{\alpha+1}}\left(\int_{0}^{1}\left|\left(\frac{b-x}{x-a}\right)^{\alpha}-\left(\frac{b-a}{x-a}-t\right)^{\alpha}\right|\right) \\
& \times\left|f^{\prime}\left(\frac{\int_{0}^{1}\left|\left(\frac{b-x}{x-a}\right)^{\alpha}-\left(\frac{b-a}{x-a}-t\right)^{\alpha}\right|\left[f^{\prime}(t x+(1-t) a)\right] d t}{\left(\int_{0}^{1}\left|\left(\frac{b-x}{x-a}\right)^{\alpha}-\left(\frac{b-a}{x-a}-t\right)^{\alpha}\right|\right) d t}\right)\right| \\
\left|I_{4}\right| \leq & \left(\gamma_{4}\right)\left|f^{\prime}\left(\frac{E x+F b}{\gamma_{4}}\right)\right| .
\end{aligned}
$$

The proof is completed.

Remark 3 On letting $\alpha=1, x=\frac{a+b}{2}$ in Theorem 6, inequality (8) reduces to inequality (4).

\section{Conclusion}

In this article, based on a more general inequality, the authors have determined a few inequalities of Hermite-Hadamard type for functions that possess a first derivative on the interior of an interval of real numbers, by utilizing the Hölder inequality and the assumptions that the mappings $\left|\left(f^{\prime}\right)\right|^{q}, q \geq 1$ are convex and concave. The outcomes exhibited here surely give refinements of those outcomes demonstrated in [1,2] and [3], and we can get many intriguing results for $\alpha=1$ and $x=\frac{a+b}{2}$. 


\section{Funding}

This research [Grant No. 5325/Federal/NRPU/R\&D/HEC/2016] was partially supported by the Higher Education

Commission of Pakistan.

\section{Competing interests}

The authors declare to have no conflict of interest.

\section{Authors' contributions}

Dr. SQ, JN and Prof. Dr. FA analyzed the problem and suggested mathematical modeling. Dr. SIB and Dr. AA generalized the results by proposing various lemmas. This manuscript has been written and revised by Prof. Dr. SH and Dr. MI, who also made some necessary corrections regarding mathematical formulations in the original. Dr. AA, Dr. MI and Prof. Dr. SH prepared a revised version of the manuscript. All authors read and approved the final manuscript.

\section{Author details}

'Department of Mathematics, COMSATS University Islamabad, Sahiwal, Pakistan. ${ }^{2}$ Department of Mathematics, COMSATS University Islamabad, Lahore, Pakistan. ${ }^{3}$ Present address: School of Mechanical and Aerospace Engineering, NANYANG Technological University, Singapore, Singapore. ${ }^{4}$ Punjab Higher Education Department (HED), Lahore, Pakistan.

${ }^{5}$ University of Engineering and Technology, Lahore, Pakistan.

\section{Publisher's Note}

Springer Nature remains neutral with regard to jurisdictional claims in published maps and institutional affiliations.

\section{Received: 11 December 2018 Accepted: 8 April 2019 Published online: 18 April 2019}

\section{References}

1. Dragomir, S.-S., Agarwal, R.-P.: Two inequalities for differentiable mapping and applications to special means of real numbers and to trapezoidal formula. Appl. Math. Lett. 11(5), 91-95 (1998)

2. Dragomir, S.-S., Chob, Y.-J., Kimc, S.-S.: Inequalities of Hadamard type for Lipschitzian mapping and their applications. J. Math. Anal. Appl. 245(2), 489-501 (2000)

3. Yang, G.-S., Hwang, D.-Y., Tseng, K.-L.: Some inequalities for differentiable convex and concave mapping. Comput. Math. Appl. 47(2-3), 207-216 (2004)

4. Beckenbach, E.-F.: Convex functions. Bull. Am. Math. Soc. 54(5), 439-460 (1948)

5. Kilbas, A.-A., Srivastava, H.-M., Trujillo, J.-J.: Theory and Applications of Fractional Differential Equations. North-Holland Mathematics Studies, vol. 204. Elsevier, Amsterdam (2006)

6. Mitrinović, D.-S., Lacković, l.-B.: Hermite and convexity. Aequ. Math. 28(1), 229-232 (1985)

7. Samet, B.: On an implicit convexity concept and some integral inequalities. J. Inequal. Appl. 2016, Article ID 308 (2016)

8. Almeida, R.-A.: Caputo fractional derivative of a function with respect to another function. Commun. Nonlinear Sci. Numer. Simul. 44, 460-481 (2017)

9. Rajba, T.: On strong delta-convexity and Hermite-Hadamard type inequalities for delta-convex functions of higher order. Math. Inequal. Appl. 18(1), 267-293 (2015)

10. Dragomir, S.-S., Bhatti, M.-I., Iqbal, M., Muddassar, M.: Some new fractional integral Hermite-Hadamard type inequalities. J. Comput. Anal. Appl. 18(4), 655-661 (2015)

11. Iqbal, M., Qaisar, S., Muddassar, M.: A short note on integral inequality of type Hermite-Hadamard through convexity. J. Comput. Anal. Appl. 21(5), 946-953 (2016)

12. Bhatti, M.-I., Iqbal, M., Dragomir, S.-S.: Some new fractional integral inequalities Hermite-Hadamard type inequalities. J. Comput. Anal. Appl. 16(4), 643-653 (2015)

13. Iqbal, M., Qaisar, S., Hussain, S.: On Simpson's type inequalities utilizing fractional integrals. J. Comput. Anal. Appl. 23(6), 1137-1145 (2016)

14. Qaisar, S., Iqbal, M., Muddassar, M.: New Hermite-Hadamard's inequalities for preinvex function via fractional integrals. J. Comput. Anal. Appl. 20(7), 1318-1328 (2016)

15. Sarikaya, M.-Z., Budak, H.: Generalized Hermite-Hadamard type integral inequalities for fractional integrals. Filomat 30(5), 1315-1326 (2016)

16. Qaisar, S., Ahmad, F., Dragomir, S.-S., I labal, M.: New Hermite-Hadamard's inequalities via fractional integrals whose absolute values of second derivatives is $P$-convex and related fractional inequalities. J. Math. Inequal. 12(3), 655-664 (2018)

17. Qaisar, S., Iqbal, M., Hussain, S., Butt, S.-I., Meraj, M.-A.: New inequalities on Hermite-Hadamard utilizing fractional integrals. Kragujev. J. Math. 42(1), 15-27 (2018)

18. Sarikaya, M.-Z., Aktan, N.: On the generalization of some integral inequalities and their applications. Math. Comput. Model. 54(9), 2175-2182 (2011) 